\title{
Síndrome Disfônica Ocupacional: Novos Aspectos desta Entidade Nosológica
}

\section{Dysphonic Occupational Syndrome: New Aspects of this Nosological Entity}

\author{
Sandra Irene Cubas de Almeida*, Paulo Pontes**. \\ * Doutora em Medicina e Coordenadora da Comissão de Foniatria da ABORLCCF. Chefe do Setor de Relações do Trabalho MTE - Gerência Norte / SP. \\ ** Professor Titular do Departamento de Otorrinolaringologia e Distúrbios da Comunicação Humana UNIFESP. \\ Instituição: Ministério do Trabalho e Emprego. \\ São Paulo / SP - Brasil. \\ Endereço para correspondência: Sandra Irene Cubas de Almeida - Avenida General Ataliba Leonel, 2764 - Parada Inglesa - São Paulo / SP - Brasil - CEP: 02242-000 \\ - Telefone: (+55 11) 2973-8927 - E-mail: sandra.almeida@mte.gov.br \\ Artigo recebido em 11 de Junho de 2010. Artigo aprovado em 28 de Junho de 2010.
}

\section{RESUMO}

Introdução:

Objetivo:

Método:

Resultados:

Conclusão:

Palavras-chave:

\section{SUMMARY}

Introduction:

Objective:

Method:

Results:

Conclusion:

Keywords:
Professores apresentam frequentemente disfunções fonatórias que se beneficiam de medidas preventivas.

Determinar a prevalência da síndrome disfônica em professores e verificar a evolução deste índice com técnicas de prevenção primária.

Foi realizado um estudo de corte transversal em professores em duas épocas distintas: na fase 1 participaram 101 professores e, na fase 2, 148 professores. A estes dois grupos foi aplicado o questionário elaborado pela Comissão Tripartite de Normatização da Voz Profissional.

A prevalência da síndrome disfônica na primeira fase foi de $70,3 \%$, enquanto que na segunda fase foi de $57,59 \%$.

$1^{\underline{a}}$ a prevalência da síndrome disfônica sofreu redução significativa com a implementação do controle médico preventivo e da organização do trabalho. $2^{\underline{a}}$ a síndrome disfônica é uma nova entidade nosológica. Estudo epidemiológico de corte transversal. Estudo de série.

disfonia, medicina preventiva, rouquidão.

Teachers often have vocal disorders that benefit from preventive measures.

To determine the prevalence of the syndrome in dysphonic teachers and check the evolution of this index with the techniques of primary prevention.

We performed a cross-sectional study of teachers in two different periods: in the first 101 teachers and participated in phase 2, 148 teachers. These two groups was administered the questionnaire prepared by the tripartite commission on regulation of the professional voice.

The prevalence of the syndrome dysphonic in the first phase was 70.3\%, while the second stage was $57.59 \%$.

1st dysphonic the prevalence of the syndrome was significantly decreased with the implementation of preventive and medical control of work organization. 2nd dysphonic syndrome is a new nosological entity. Cross-sectional epidemiological study. Study series.

dysphonia, preventive medicine, hoarseness. 


\section{INTRODUÇÃO}

Professores de todos os níveis de ensino necessitam de um eficaz funcionamento do sistema fonatório para o exercício de sua profissão. As disfunções fonatórias que se desenvolvem ou exacerbam-se no decorrer de sua atividade, manifestam-se, inicialmente, por sintomas e sinais, cuja causa atribui-se ao uso profissional da voz associado ou não a outros fatores.

Em decorrência da diversidade sintomatológica e da causalidade múltipla nos transtornos da voz propomos designar o conjunto de fatores relacionados à perda de saúde vocal pelo uso profissional de Síndrome Disfônica Ocupacional (SDO).

Com base no conceito de saúde clássico, podemos afirmar que a saúde vocal representa o estado do equilíbrio entre a forma (sistema fonatório) e a função (fonação), de um lado, e a ação das forças que tendem a perturbá-los, de outro lado. Este inter-relacionamento não é passivo, é uma resposta ativa do organismo no sentido de se adaptar às exigências. Entre a adaptação e a não adaptação ao uso profissional da voz existe uma graduação que se estende da eufonia com aqueles que se sentem em excelentes condições, passando pelos diferentes graus de disfonias que vão dos que estão razoavelmente bem aos que se sentem abaixo do normal até os que realmente estão incapacitados.

Segundo Leavell \& Clark (1), a história natural de qualquer processo mórbido no ser humano apresenta dois períodos nos quais há o desenvolvimento e instalação da doença: pré-patogênico e patogênico.

No período pré-patogênico há a atuação dos fatores de risco ambientais no indivíduo que apresenta predisposição aos agressores.

O período patogênico compreende as fases de: patogenia precoce, que precede a da precoce discernível, seguidas pelas fases da patogenia avançada e a da convalescença.

A Síndrome Disfônica Ocupacional tem sua aplicação conceitual nas fases precoces de patogenia da doença vocal funcional ou orgânica.

Transpondo este conceito para a SDO, no período pré-patogênico três fatores se inter-relacionam, ou seja, os individuais como sexo, idade e inadaptações fônicas, os ambientais como ruído, acústica, uso de microfones, organização do trabalho, ergonomia e estresse da atividade e os pré-existentes como distúrbios hormonais, processos infla- matórios de vias aéreas, síndrome faringo-laríngea do refluxo, auto-medicação, tabagismo, alcoolismo e ingestão de cafeína.

O período patogênico precoce se inicia no momento em que o fator desencadeante atua e o hospedeiro (o profissional da voz) não se adapta e responde com modificações funcionais ou nos tecidos no sistema fonatório. Estas alterações não são evidentes pelos métodos atuais diagnósticos e, necessitam de critérios de conhecimento clínico que considerem a história natural da doença desde os seus primórdios,ou seja,do período pré-patogênico e patogênico precoce.

Nestas fases, tem-se a possibilidade de reversão da doença.

No processo evolutivo do distúrbio começam a surgir os sintomas que são reversíveis ao se retirar o agente, o que configura a patogênese precoce discernível e que ainda não impede o pleno desenvolvimento da atividade. Quando evolui para o impedimento parcial da atividade inicia-se a fase da patogênese avançada e necessita de tratamento; outro marco que podemos acrescentar para definir a fase avançada é o aparecimento de lesões na laringe, que inserido na SDO designamos de Laringopatia Ocupacional (LO) (2).

Em caso de manutenção das alterações (cronicidade) ou suas consequências extremas na convalescença podemos atingir o grau de disfonia de invalidez definitiva.

Com o objetivo de evitarmos a incapacitação, temos que interferir nos fatores nocivos detectáveis do período pré-patogênico ao patogênico precoce discernível.

As técnicas de prevenção primária devem, então, serem aplicadas, evitando o afastamento do profissional de suas atividades e, revertendo o desenvolvimento da doença. Com medidas preventivas, visamos manter a saúde vocal pela proteção específica do professor e pelo estabelecimento de barreiras contra os agentes do meio-ambiente.

No período pré-patogênico e na fase de patogênese precoce dificilmente identificamos os profissionais da $\mathrm{VOZ}$ que potencialmente irão passar para as fases seguintes através dos meios de diagnóstico convencionais, pois, todos estão voltados para a presença de lesão orgânica ou funcional instalada. Da fase de patogenia avançada para diante, pela incapacitação profissional, os próprios profissionais se identificam pela necessidade do diagnóstico e cuidados. Objetivando a prevenção da invalidez é essencial encontrarmos aqueles que estão na fase de patogênese precoce discernível, assim como no período pré-patogêncio. Este grupo, por possuir condições de se manter em 
atividade, não se apresenta espontaneamente, porém pode ser identificado pela busca de sintomas e o levantamento das condições de trabalho. Na fase de patogênese avançada o profissional exigirá cuidados de prevenção secundária com técnicas direcionadas e personalizadas e essencialmente habilitatórias; geralmente há afastamento temporário de suas atividades, mas com possibilidade plena de retorno. Na convalescença agimos com prevenção terciária com ênfase na reabilitação com possibilidade duvidosa de retorno.

Considerando estes aspectos realizamos, por meio do Programa de Prevenção da Disfonia Ocupacional em Professores Universitários da Zona Norte de São Paulo, um estudo com o objetivo de determinar a prevalência da Síndrome Disfônica Ocupacional e verificar a evolução deste índice após a introdução de técnicas de prevenção primária.

\section{MÉTOdo}

Realizamos um estudo epidemiológico de corte transversal com a participação voluntária de professores de uma Instituição Universitária da região norte da cidade de São Paulo. Este estudo foi executado em duas épocas distintas: 2001 (fase 1) e 2004 (fase 2). Recebeu a aprovação do Comitê de Ética Médica e teve a assinatura no Termo Esclarecido de Consentimento dos professores e dos responsáveis pela Instituição. Foi conduzido por equipe médica acompanhados por representantes do Sindicato dos trabalhadores.

A fim de cumprir o objetivo de determinar a prevalência da fase de patogênese discernível da SDO, utilizamos o questionário de auto-avaliação elaborado pela Comissão Tripartite (3) conforme Portaria 253 que foi publicada no Diário Oficial da União em 31 de julho de 2002. Deste questionário retiramos os dados relativos a sintomas que podem ser encontrados em disfonias como: dor ou irritação na garganta (S1), sensação de corpo estranho (S2), necessidade de pigarrear (S3), dor cervical (S4) e rouquidão (S5) subdividida em constante, constante com flutuações e intermitente.

Os sintomas foram analisados comparativamente de forma individualizada e também em associação (quando dois ou mais presentes), esta caracterizando a SDO.

Os questionários foram entregues aos professores que se encontravam na sala dos professores nos períodos que precediam as aulas no início dos anos letivos 2001 e 2004, sendo voluntárias as participações e sem a identificação pessoal nas respostas. No período de 2001 a 2004 foram apresentadas à Instituição e aos professores em geral medidas preventivas abrangendo: estímulo à hidratação, proteção contra a inalação de substâncias irritantes da mucosa tais como o tabaco e poeiras, contra-indicação à auto-medicação e consumo de cafeína, correção dos desvios posturais, conceitos do uso incorreto e abusivo da voz e orientação quanto ao repouso vocal.

Foram analisadas em porcentagem as prevalências quanto aos sintomas isolados e quanto aos sintomas associados, isto é, dois ou mais, para caracterizar a SDO.

Foram obtidos dados relativos à idade e ao gênero a fim de se constatar a equivalência da constituição dos grupos nas fases 1 e 2 .

A análise estatística foi realizada para a verificação da associação entre as variáveis dependentes com as variáveis independentes (fase 1 e fase 2) aplicando-se testes não paramétricos de associação Qui-quadrado ou teste exato de Fisher. Fixou-se o nível de significância de $5 \%(\mathrm{p} \leq 0.05)$. O banco de dados foi criado no Excel e para as análises utilizou-se o Software SAS /SAT versão 9.12.

\section{RESUltAdOS}

A média de idade foi de 40.86 anos com desvio padrão de 8.9 anos, mínima de 29 anos e máxima de 86 anos na fase 1, com participação de 101 professores.

Na fase 2, a média de idade foi de 38.23 anos, com desvio padrão de 8.32 anos, mínima de 34 anos e máxima de 64 anos, com participação de 148 professores.

Nos Gráficos 1 e 2 são apresentadas em porcentagem as prevalências quanto aos sintomas isolados e quanto aos sintomas associados (SDO).

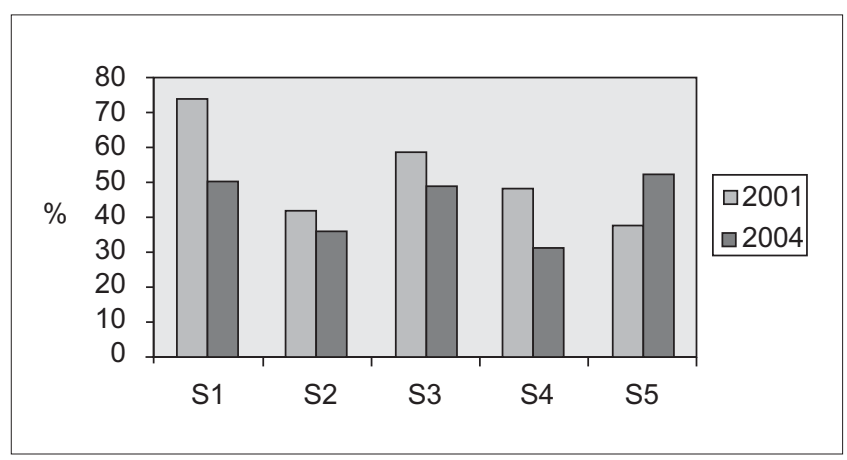

Gráfico 1. Evolução da prevalência em porcentagem dos sintomas da Síndrome Disfônica Ocupacional da fase 1 para a fase 2. $-\mathrm{S} 1=$ dor ou irritação $\mathrm{p}=0,001 \mathrm{~S} 2=\operatorname{sensação~de~}$ corpo estranho $\mathrm{p}=0,2 \mathrm{~S} 3=$ necessidade de pigarrear $\mathrm{p}=0,05$ $\mathrm{S} 4=$ dor cervical $\mathrm{p}=0,008 \mathrm{~S} 5=$ rouquidão $\mathrm{p}=0,20$. 


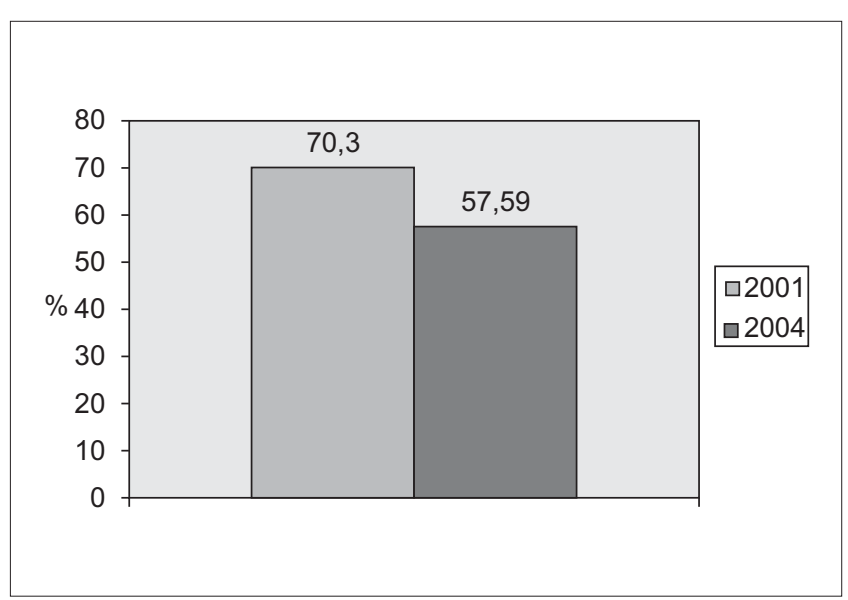

Gráfico 2. Evolução da prevalência em porcentagem na Síndrome Disfônica Ocupacional da fase 1 para a fase 2 . $-\mathrm{p}=0,05$.

No Gráfico 3 temos as três modalidades do sintoma rouquidão.

\section{DISCUSSÃO}

A influência de variáveis não ponderadas na resposta ao questionário foi controlada através da metodologia empregada na sua aplicação, pois o mesmo era distribuído e recolhido no mesmo ato do contato com os professores, fazendo com que as respostas tivessem um caráter espontâneo imediato e reduzindo a abstinência à participação. $\mathrm{O}$ fator espontaneidade, assim como a garantia de preservação das informações sem a necessidade de identificação do participante na coleta das respostas, foi destacado durante todo o trabalho.

Os processos de saúde ou doença do trato vocal dependem da preservação da sua estrutura celular. Neste ponto encontramos a confluência entre os estudos morfohistológicos e o controle epidemiológico da Síndrome Disfônica Ocupacional, pois o sub-aproveitamento ou estado não ótimo do substrato tecidual responde pelo desencadeamento dos sintomas até a evolução para a laringopatia ocupacional.

A queixa de dor ou irritação na garganta foi o sintoma mais comum relatado na fase 1 do estudo, seguido pelo sintoma de necessidade de pigarrear, com $74,2 \%$ e $58,5 \%$, respectivamente. Com a introdução das medidas preventivas tivemos redução significativa para $50,5 \%$ e $49,0 \%$. A rouquidão, aqui referida como toda alteração na qualidade da emissão vocal, foi referida fundamentalmente como intermitente, ou seja, há períodos de maior exigência do desempenho vocal e/ou os fatores que atuam negativamente se exacerbam. De todos os sintomas foi o de menor prevalência na fase 1 ,

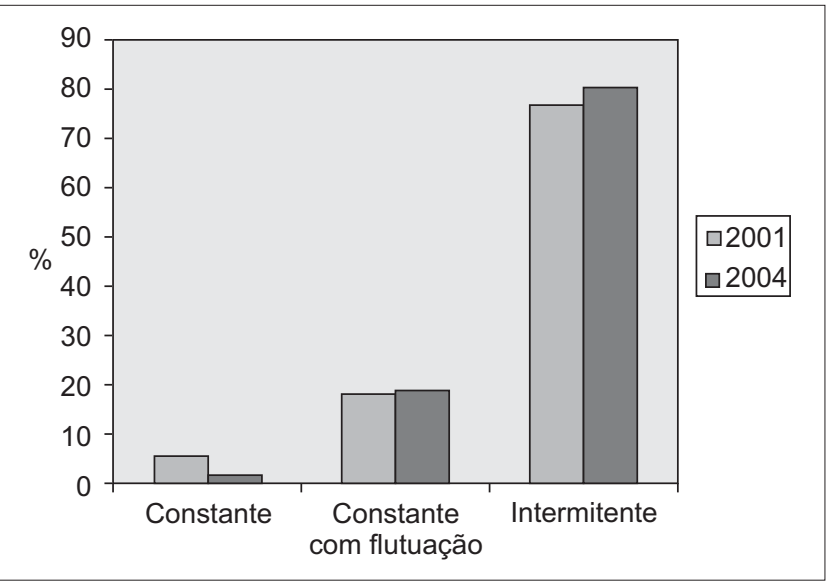

Gráfico 3. Prevalência das modalidades da rouquidão, em porcentagem, na Síndrome Disfônica Ocupacional da fase 1 para a fase 2 .

isto é, $37,2 \%$. Na fase 2, diferentemente dos demais sintomas, a prevalência aumentou de forma significativa para 52,3\%. Este resultado, que parece ser paradoxal, pode ser explicado pelo fato de ser obtido por intermédio do questionário de auto-avaliação; os professores, ao serem orientados e alertados para o risco vocal adquiriram aumento de sua capacidade perceptiva auditiva além de adquirirem uma atitude mais criteriosa frente à própria vOZ.

A dor cervical com prevalência na fase 1 de $47,4 \%$ e a de sensação de corpo estranho com 41,8\% estão mais relacionadas à tensão e ao esforço muscular, bem como a distúrbios posturais. Dos dois, a dor cervical teve redução significativa na fase $2 \mathrm{com} 30,9 \%$. Podemos explicar esta queda pela intervenção no ambiente de trabalho com análise e planejamento ergonômicos que atuaram adequando a postura corporal à tarefa a ser executada; a Instituição foi orientada quanto ao uso das cadeiras e mobiliário para repouso. Quanto à sensação de corpo estranho a redução não significante expressa a influência de outras causas além do uso da voz na Síndrome Disfônica Ocupacional, como a síndrome faringo-laríngea do refluxo, tensão emocional, etc.

A prevalência da Síndrome Disfônica Ocupacional considerando-se a fase patogênica precoce discernível foi de $70,3 \%$, o que corresponde aos dados da literatura quando é pesquisada sob a denominação genérica de disfonia ocupacional. Com o tratamento preventivo primário ocorreu redução significativa para 57,5\%.

Não sabemos o quanto esta prevenção atuou nos que estavam na fase de patogenia precoce e que se beneficiaram; provavelmente este número foi superior ao da fase discernível pois estavam menos afetados e teoricamente mais vulneráveis às medidas de proteção. 
Da análise dos nossos resultados fica evidenciado que a atuação preventiva primária nos estabelecimentos de ensino pode reduzir de forma significativa a fase de patogenia precoce discernível que precede a de patogenia avançada em todos os inconvenientes dos afastamentos do trabalho, que prejudicam o professor, o Corpo Dicente e a Instituição.

\section{CONCLUSÃO}

A prevalência da fase patogênica discernível da Síndrome Disfônica Ocupacional ocorreu na maioria dos professores universitários e sofreu redução significativa com o controle médico preventivo e da organização do trabalho.

A Síndrome Disfônica Ocupacional é uma nova entidade nosológica cuja importância clínica do diagnós- tico interfere na história natural das laringopatias orgânicas.

\section{REFERÊNCIAS BIBLIOGRÁFICAS}

1. Leavell H, Clark EG. Medicina Preventiva. 1 Ed. São Paulo: McGrawHill do Brasil; 1976.

2. $3^{\circ}$ Consenso Nacional Sobre Voz Profissional. Rio de Janeiro. 2004.

3. Comissão Tripartite de Normatização para a Voz Profissional. Ministério do Trabalho e Emprego. Questionário de auto-avaliação para professores. São Paulo: Imprensa Oficial do Estado; 2002. 